\title{
In situ observation of electric field induced agglomeration of carbon black in epoxy resin
}

\author{
Torsten Prasse \\ Microelectronic Materials Section, Technical University Hamburg-Harburg, D-21071 Hamburg, Germany \\ Lionel Flandin \\ CERMAV-CNRS, Université Joseph Fourier, BP 53, 38041 Grenoble Cedex 9, France \\ Karl Schulte \\ Polymer/Composites Section, Technical University Hamburg-Harburg, D-21071 Hamburg, Germany \\ Wolfgang Bauhofer ${ }^{\mathrm{a})}$ \\ Microelectronic Materials Section, Technical University Hamburg-Harburg, D-21071 Hamburg, Germany
}

(Received 5 February 1998; accepted for publication 26 March 1998)

\begin{abstract}
This letter reports on the influence of a static electric field applied by metal electrodes on the agglomeration process of carbon black $(\mathrm{CB})$ in epoxy resin. The growth of dendrites from the anode into the material is observed in situ by optical transmission microscopy. A percolating network is seen to form, combined with a drastic reduction in the sample resistivity. This behavior can be explained by taking into account the electrostatic interaction of the charged CB particles. The final resistance for composites with a given $\mathrm{CB}$ content can be controlled within a range of several decades by varying the applied voltage and the curing temperature of the mixture. (C) 1998 American Institute of Physics. [S0003-6951(98)01622-2]
\end{abstract}

The use of composites made from an insulating matrix and highly conductive fillers is becoming more and more important due to their ability to electromagnetically shield and prevent electrostatic charging of electronic devices. A low filler volume fraction is required to maintain the mechanical properties of the matrix. In recent years, different models have been proposed ${ }^{1,2}$ to explain the formation of a conductive filler network.

Statistical percolation is an established theory which is suitable for randomly dispersed but not for interacting fillers. ${ }^{3,4}$ In three-dimensional systems this theory predicts a percolation volume fraction $\left(V_{c}\right)$ of $16 \%$ to form a continuous filler network. ${ }^{4}$ Nevertheless, it has been demonstrated in a number of investigated materials that the particles are not randomly dispersed ${ }^{1,5-8}$ and that the experimental data do not correspond with the predictions of the standard percolation model.

The agglomeration process of carbon black (CB) dispersed in epoxy resin is described using a model based on the colloid theory by Schueler et al. ${ }^{8,9}$ This theory assumes that the CB particles are charged during the preparation process. Thus, an electrostatic particle-particle interaction has to be taken into account. There are two competitive forces, namely, the short-range van der Waals attraction and the long-range Coulomb repulsion. Below the critical volume fraction of $0.9 \%$ the Coulomb repulsion prevents the formation of clusters in the bulk of the material which means that the dispersion is stabilized electrostatically. The particles move away from each other to reduce their interaction potential energy. For particle volume fractions above the critical value, there is a sharp rise in the conductivity. This is due

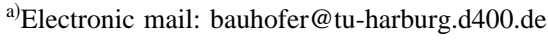

to a transition from a dispersed to an agglomerated state of the CB particles.

The aim of the present work is to obtain direct evidence for the charge on the $\mathrm{CB}$ particles. Therefore a microscopic observation method was used for direct imaging of the agglomeration process controlled by an applied electric field.

The matrix used in this study is an epoxy polymer based on bisphenol-A resin (ARALDITE LY556, CIBA GEIGY) and an aromatic amine hardener (ARALDITE HY932). The conductive filler component was carbon black (PRINTEX XE2, DEGUSSA AG) with a primary particle diameter of about $30 \mathrm{~nm}$. The sample preparation before curing has already been described elsewhere. ${ }^{8,9}$ In contrast to customary preparation methods, the CB-epoxy dispersion was stirred for one hour at a high speed of $5000 \mathrm{rpm}$. The hardener was then added and the composite mixed again for one hour at $2000 \mathrm{rpm}$.

Glass slides with sputtered silver strip electrodes and an electrode spacing of $500 \mu \mathrm{m}$ were used for the microscopic investigations. The CB-epoxy mixture was dispersed between the electrodes. A KEITHLEY 617 electrometer was used to record the current measurements. Two thirds of the initial value of the current (see Fig. 1) can be attributed to leakage whilst the remainder is due to the residual conductivity of the epoxy resin. To monitor the resistance during the curing process, the voltage was applied by means of polished brass parallel plates dipped into the dispersion.

Figure 1 shows the plot of the current versus time during curing of the $0.12 \% \mathrm{CB}$-epoxy dispersion used for the optical microscopy. A voltage of $30 \mathrm{~V}$ was applied between the strip electrodes. This voltage results in an electric field strength of $600 \mathrm{~V} / \mathrm{cm}$ which forces the particles to move towards the electrodes. At the beginning of the curing process the $\mathrm{CB}$ is evenly distributed, and no cluster formation 


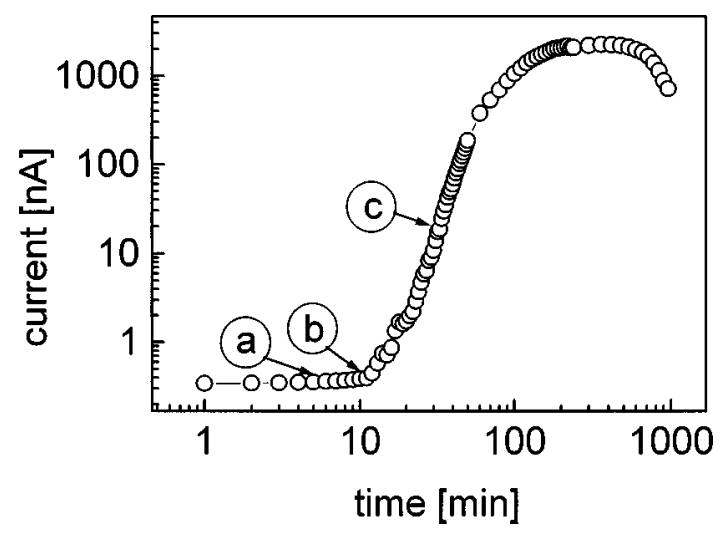

FIG. 1. Plot of the electric current versus time for a CB content of $0.12 \%$ and an applied voltage of $30 \mathrm{~V}$.

appears equivalent to the right-hand side of the micrograph (a) in Fig. 2. After about 5 min the first clusters start to appear at the anode [see left-hand side of Fig. 2(a)] indicating a negative charge on the $\mathrm{CB}$. The current remains at its initial value. A percolating cluster forms after $12 \mathrm{~min}$ [Fig. 2(b) ] causing a sharp increase in the measured current. Figure 2(c) shows the network after a duration of $40 \mathrm{~min}$. It is obvious that the continuing process of network formation is linked with a still rising sample current. After about $100 \mathrm{~min}$ of curing the viscosity of the epoxy resin increases and the network becomes fixed with the current reaching its maxi-
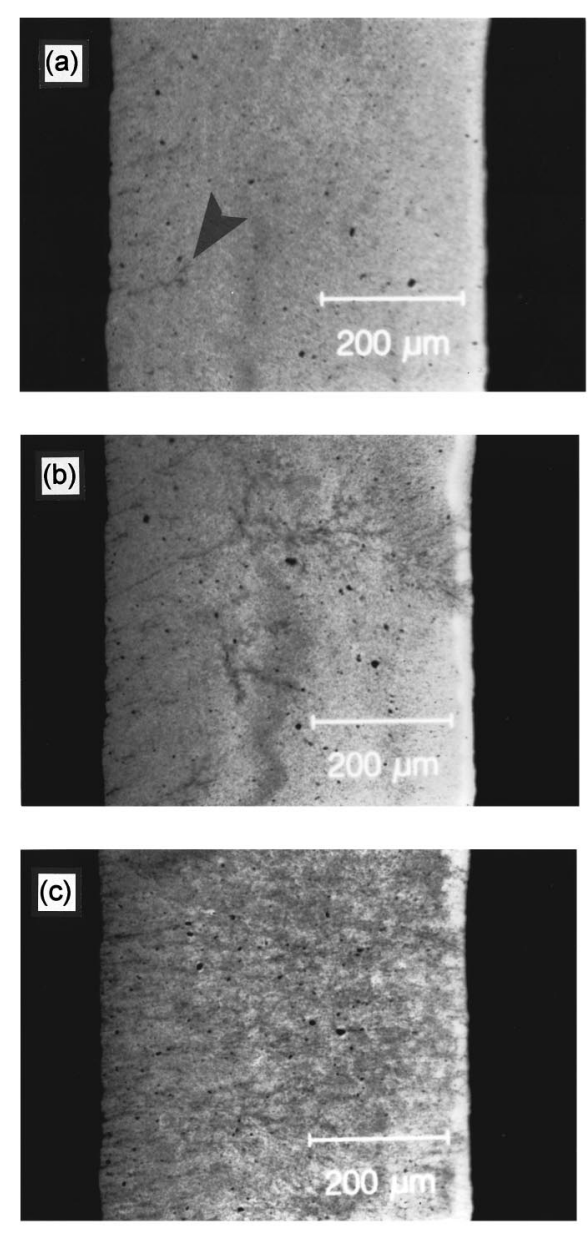

FIG. 2. Transmission optical micrographs taken after 5, 12, and 40 min corresponding to the positions denoted by (a), (b), (c) in Fig. 1.

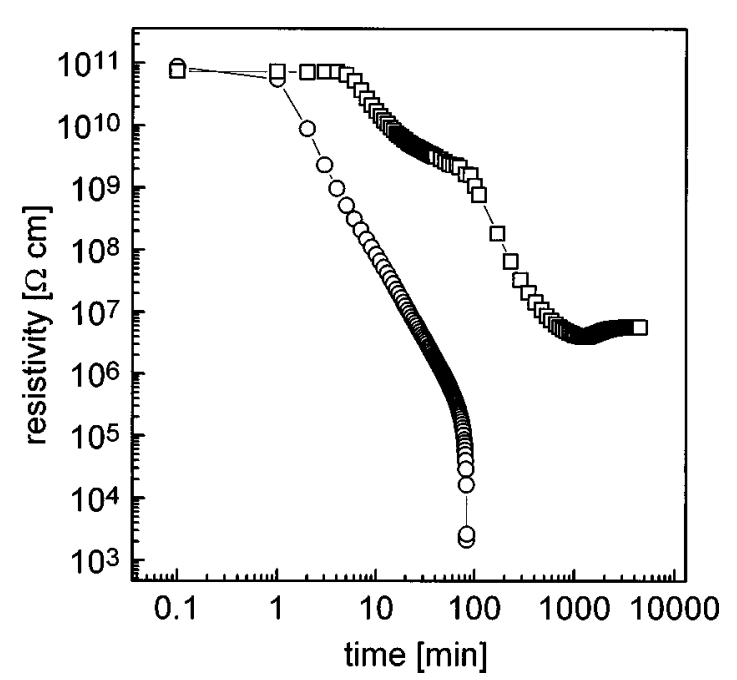

FIG. 3. Time dependence of the electrical resistivity for a $\mathrm{CB}$ content of $0.6 \%$ and applied voltages of $30 \mathrm{~V}(\square)$ and $60 \mathrm{~V}(\bigcirc)$.

mum which is about four orders of magnitude higher than the initial value.

Figure 3 shows the time dependence of the sample resistivity at room temperature for a content of $0.6 \% \mathrm{CB}$. Voltages of 30 and $60 \mathrm{~V}$ were applied according to electric field strengths of 50 and $100 \mathrm{~V} / \mathrm{cm}$. The initial resistance of the samples can be attributed to the residual conductivity of the epoxy resin. ${ }^{9}$ In the $30 \mathrm{~V}$ curve this resistance remains constant up to $5 \mathrm{~min}$ after applying the voltage. Then there is a decrease due to the formation of a percolating network of CB particles which is consistent with the microscopical observations. After about $300 \mathrm{~min}$ the sample hardens and the resistivity reaches its minimum value. There is an equal initial resistance in the curve with an applied voltage of $60 \mathrm{~V}$ but after just one minute the resistivity begins to decrease indicating a quicker formation of the percolating network. After about $60 \mathrm{~min}$ the sample current reaches a value of $2 \mathrm{~mA}$ which is sufficient to heat the sample. The temperature increase reduces the viscosity of the epoxy resin and the CB particles move and aggregate rapidly which further decreases the resistivity. Due to this positive feedback phenomenon the agglomeration process deviates from equilibrium and shows an "avalanche" behavior. A comparison with the results of Schueler et al. ${ }^{8}$ indicates that at the final resistivity of 2000 $\Omega \mathrm{cm}$ almost all CB particles are aggregated onto the network.

Figure 4 shows the resistivity versus the curing time at an applied voltage of $30 \mathrm{~V}$ for the two temperatures 28 and $38^{\circ} \mathrm{C}$. CIBA GEIGY ${ }^{10}$ reports a drop in the viscosity of about $60 \%$ in this temperature range before curing. The $28{ }^{\circ} \mathrm{C}$ curve is equivalent to the one described above for 30 $\mathrm{V}$ applied voltage. In the curve with a resin temperature of $38^{\circ} \mathrm{C}$ we observe a lower initial resistivity of the epoxy resin. There is a sudden decrease in the resistivity which indicates the rapid formation of a percolating network. This illustrates the considerable influence of temperature and thus viscosity on the agglomeration dynamics of the $\mathrm{CB}$ particles. Hardening occurs earlier in the $38^{\circ} \mathrm{C}$ curve than in the $28{ }^{\circ} \mathrm{C}$ curve due to the quicker curing of the sample. Nevertheless, the $38{ }^{\circ} \mathrm{C}$ curve reaches a resistance value which is about 100 times smaller. In both cases the agglomeration process, 


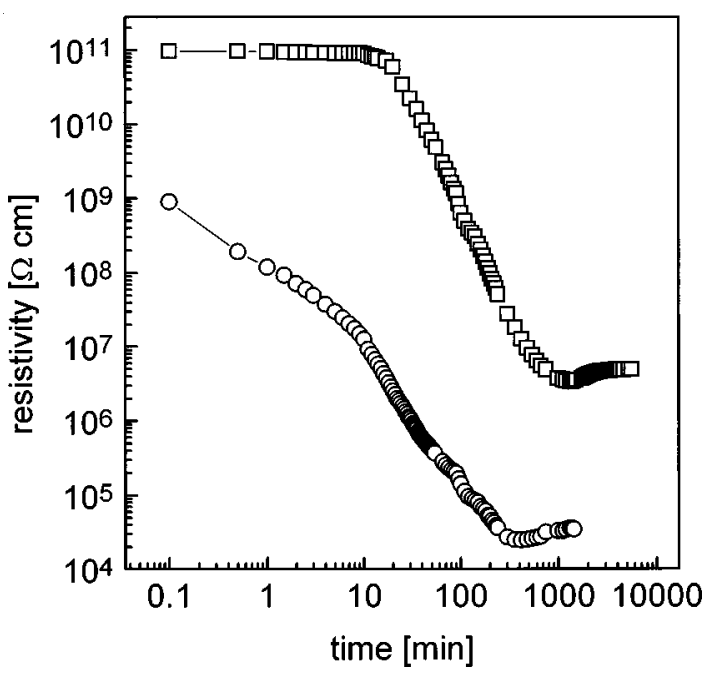

FIG. 4. Time dependence of the electrical resistivity at temperatures of $28^{\circ} \mathrm{C}(\square)$ and $38^{\circ} \mathrm{C}(\bigcirc)$ for samples with an amount of $0.6 \% \mathrm{CB}$ and an applied voltage of $30 \mathrm{~V}$.

and thus the final resistivity, are limited by the onset of hardening; this becomes evident when the final resistivities are compared with those of the $60 \mathrm{~V}$ curve in Fig. 3.

The experimental results can be explained by considering the interaction between the charged particles and the external electric field. Assuming a CB concentration below the critical value of $0.9 \%$ due to the electrostatic repulsion of the particles, no cluster formation takes place without applying an electric voltage. By applying a voltage to the electrodes, the negatively charged particles move to the anode and discharge there. Further particles drain off their charge directly at the tips of the $\mathrm{CB}$ clusters, i.e., the points of the highest electric field strengths. Dendrites grow to the volume of the material. With the formation of the first percolating pathways a characteristic increase in the measured electronic current occurs. Where there are no curing restrictions all $\mathrm{CB}$ particles are incorporated in the network. This applies to the CB rapidly agglomerated by current heating of the sample. The dynamics of the agglomeration process can be described as a sensitive balance which depends on the electrostatic interaction of the particles with the external electric field and the viscosity of the epoxy matrix. This process is considerably influenced by the sample preparation, the value of the applied voltage and the curing temperature.

In conclusion, a new method for the preparation of conductive CB-epoxy composites with a low CB content of $0.12 \%$ has been reported. The method allows adjustment of the final resistivity for a composite with a given CB volume fraction within a range of about seven decades by varying the applied voltage. The growth process of the CB clusters was verified in situ by optical microscopy. In addition, we point out the possibility of preparing a material with a spatial inhomogeneous conductivity by applying the voltage to patterned electrodes during the curing process.

The authors wish to mention the untimely death of Dr. R. Schueler on whose doctoral thesis this work was based. They wish to thank the DAAD-PROCOPE research collaboration program for its financial support.

${ }^{1}$ F. Lux, J. Mater. Sci. 28, 285 (1993).

${ }^{2}$ F. Carmona, Physica A 157, 461 (1989).

${ }^{3}$ S. Kirkpatrick, Rev. Mod. Phys. 45, 574 (1973).

${ }^{4}$ R. Zallen, The Physics of Amorphous Solids (Wiley, New York, 1983).

${ }^{5}$ M. Sumita, H. Abe, H. Kayaki, and K. Miyasaka, J. Macromol. Sci., Phys. B25, 171 (1986).

${ }^{6}$ M. B. Heaney, Phys. Rev. B 52, 12477 (1995).

${ }^{7}$ L. J. Adriaanse, J. A. Reedijk, P. A. A. Teunissen, H. B. Brom, M. A. J. Michels, and J. C. M. Brokken-Zijp, Phys. Rev. Lett. 78, 1755 (1997).

${ }^{8}$ R. Schueler, J. Petermann, K. Schulte, and H.-P. Wentzel, J. Appl. Polym. Sci. 63, 1741 (1997).

${ }^{9}$ R. Schueler, Ph.D. thesis, Technical University Hamburg-Harburg, 1994. ${ }^{10}$ CIBA-GEIGY, Publ. Nr. 24668/d. 